\title{
Androgen insensitivity syndrome: discussion based on three cases
}

\section{Gaurav Sharma*, Rita Ghode}

Department of Radio diagnosis, Datta Meghe Institute of Medical Sciences, JNMC Sawangi (Meghe), Wardha Maharashtra

Received: 18 July 2015

Accepted: 14 August 2015

\author{
*Correspondence: \\ Gaurav Sharma, \\ E-mail: drgsharma78@gmail.com
}

Copyright: ( $\odot$ the author(s), publisher and licensee Medip Academy. This is an open-access article distributed under the terms of the Creative Commons Attribution Non-Commercial License, which permits unrestricted non-commercial use, distribution, and reproduction in any medium, provided the original work is properly cited.

\begin{abstract}
Androgen insensitivity syndrome is an X-linked recessive condition due to a complete or partial insensitivity to androgen, resulting in a failure of normal masculinisation of the external genitalia in chromosomally male individuals. This failure of virilisation can be either complete or partial depending on the amount of residual androgen receptor function. It is classified into three categories depending upon the degree of genital masculinisation into complete, partial and mild insensitivity with phenotype variation from normal female at one end to normal male on other end. Here we described two cases of partial and one case of complete androgen insensitivity syndrome. The presenting feature in partial androgen insensitivity patients was amenorrhea in 16 year female and ambiguous genitalia in 7 months baby. The complete androgen insensitivity case presented with inguinal hernia only at 6 years of age. All the three cases were classified according to Quigley scale with Karyotyping confirming 46 XY genotype. After full evaluation two patients had undergone gonadectomy while in third case parents opted to take definitive decision in future. The role of psychological support and counselling is also emphasized with timing of disease disclosure to patient left over to the parents for appropriate age.
\end{abstract}

Keywords: Ambiguous genitalia, Inguinal hernia, Testicular feminisation syndrome

\section{INTRODUCTION}

Androgen insensitivity syndrome is a $\mathrm{X}$-linked recessive disorder of sex development known collectively as 46 XY DSD characterized by variable defects in virilisation of genotypical 46XY individual secondary to mutations in Androgen Receptor gene with resultant end organ insensitivity to androgen action influencing both the morphogenesis and differentiation of the body structure and systems in which this hormone exerts its effects. ${ }^{1}$ Hormone resistance syndrome was first described by Dr. John Morris who coined the term testicular feminisation syndrome. ${ }^{2}$ Androgen insensitivity syndrome (AIS) is said to be complete when there is no response to androgen with typical female phenotype. Partial androgen insensitivity syndrome occurs when androgen receptors can partially respond to androgen stimulation with partial masculinisation of external genitalia. Mild androgen insensitivity syndrome is indicated with normal male external genitalia. The aim of this paper is to present the varied clinical manifestations of AIS and the important role of imaging in early diagnosis of cases presented with inguinal hernia alone by describing three cases, one case of complete androgen insensitivity syndrome and two cases of partial androgen insensitivity syndrome.

\section{CASE REPORT}

Our first patient was 6 years old female presented in surgical outpatient clinic with swelling in right inguinal region. On Local examination right inguinal hernia was confirmed. Patient was referred for Ultrasonography to evaluate further. Ultrasound revealed right inguinal hernia with testis a like structure within the sac with another testicular structure in left inguinal canal. Pelvic scan showed absent uterus and ovaries. 
MRI scan confirmed uterocervical agenesis, absent ovaries with blind ending short vagina. Presence of ectopic gonads was also confirmed, with right testis in deep inguinal ring and left testis near left superficial inguinal ring (Figure $1 \mathrm{~B}$ and $\mathrm{C}$ ). Prospective local examination showed normal female genitalia without any ambiguity (Figure $1 \mathrm{~A}$ ). Parents were explained for the possible diagnosis of androgen insensitivity syndrome and after consent Karyotyping was done which confirmed the $46 \mathrm{XY}$ genotype making diagnosis of complete androgen insensitivity syndrome (CAIS). With due parent consent gonadectomy was performed with hernia repair. Parents were fully explained regarding need of oestrogen therapy near puberty and choice is left over to parents to slowly reveal about the disease to child at appropriate age.

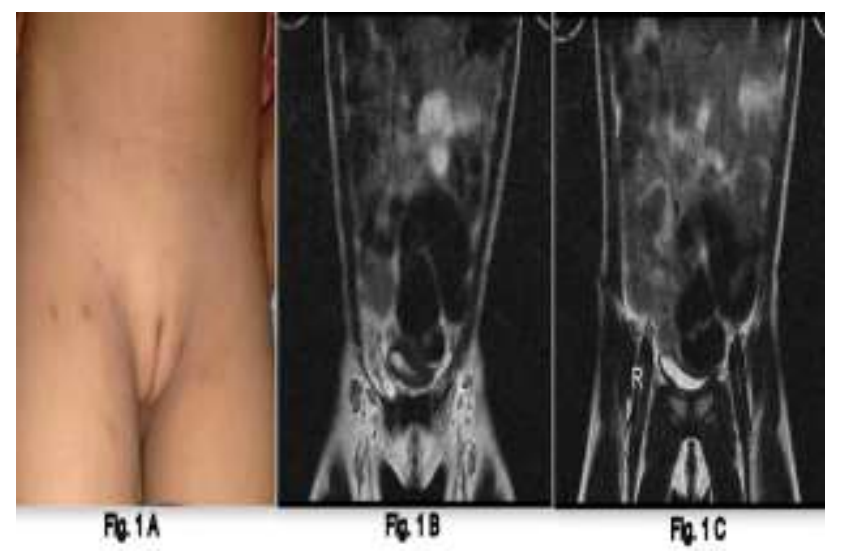

Figure 1(A): Clinical photograph with normal appearing external genitalia; $(B)$ coronal $\mathrm{T} 2$ weighted MR images localising left testis $(L)$ in the left inguinal canal near left superficial inguinal ring; (C) coronal T2 weighted MR image showing right testis $(R)$ lying just distal to right deep inguinal ring by the side of bladder.

Second case was 16 years old female presenting with primary amenorrhea and swelling over right labial region. Physical examination showed coarsened facial features, average height $165 \mathrm{~cm}$, age appropriate breast development (Tanner IV) and sparsed axillary hairs. External genitalia was ambiguous with microphallous, clitoromegaly, fused labia, right labial swelling with presence of pubic hairs (Figure 2A). Per vaginal examination revealed $3 \mathrm{~cm}$ long blind ending vagina with absent cervix. Both USG and MRI studies confirmed agenesis of uterus, cervix, and bilateral ovaries with short vagina. Right labial swelling had ectopic right testis measuring $3.4 \times 1.5 \mathrm{~cm}$, left testis localised in left mid inguinal canal of size $3.1 \times 1.5 \mathrm{~cm}$ (Figure $2 \mathrm{~B}$ and $\mathrm{C}$ ). Imaging studies also confirmed presence of penile root with corpora cavernosa and spongiosa.

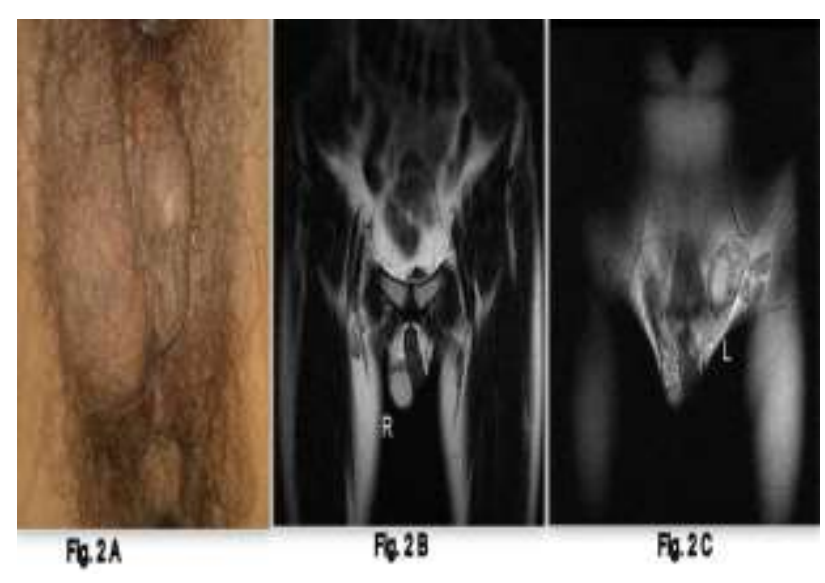

Figure 2(A): Clinical photograph with partial AIS grade 4 ambiguous genitaia with microphallus structure like clitoromegaly, partially fused labia, swollen right labia containing ectopic right testis; (B) coronal T2 weighted MR image showing homogeneous intermediate signal intensity right undescended testis within enlarged right labia and microphallus like structure opening commonly with urethra; (C) Coronal T2 weighted MR image showing ectopic left testis in mid left inguinal canal.
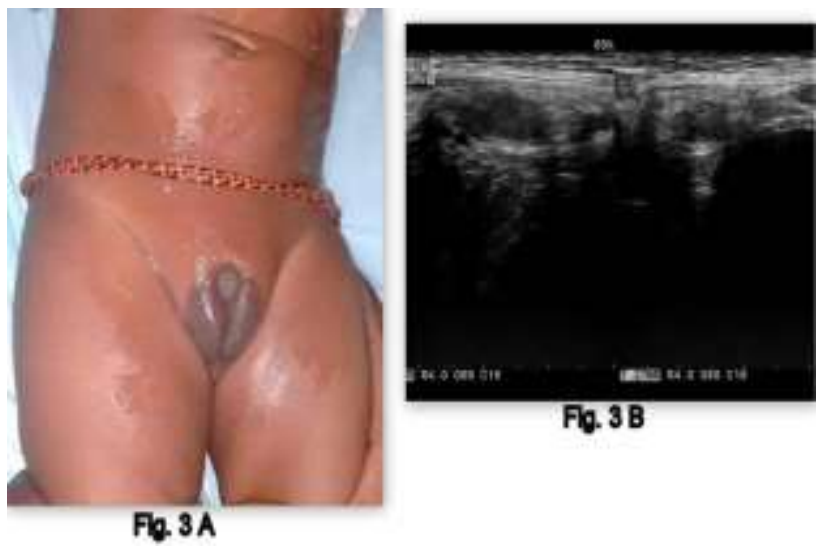

Figure 3(A): Clinical photograph with grade 3 partial AIS ambiguous genitalia with bifid scrotum, micropenis and hypospadias; (B) ultrasound image showing bilateral ectopic testis in mid inguinal canal.

Hormonal levels were in normal range: testosterone 12.2 nmol/l; DHT 1.3 nmol/l; FSH 8 mIU/ml; LH 6.2 mIU/ml; estrogen $66 \mathrm{pg} / \mathrm{ml}$. Karyotyping revealed $46 \mathrm{XY}$ genotype making final diagnosis of Partial androgen insensitivity syndrome. Patient's parents counselled about the disease and treatment option. As the case was raised and nurtured as a girl, they choose to be the girl lifelong and hence bilateral orchidectomy was done under consent with oestrogen supplements.

Our third case was 7 months female baby presented in paediatrics clinics with ambiguous genitalia. Local examination revealed bifid scrotum with micropenis and perineoscrotal hypospadias (Fig 3A). Ultrasonography showed absence of uterus, cervix, and both ovaries. 
Ectopic testes were located in bilateral mid inguinal canal (Figure 3B). After explaining the possible diagnosis of disorder of sex development (PAIS) and consent, Karyotyping performed which revealed 46 XY genotype.

Parents were explained for the need for gonadectomy and repair of hypospadias with option to choose the phenotype. Parents decided to take appropriate decision at latter half of life.

\section{DISCUSSION}

Androgen insensitivity syndrome (AIS) is the most frequent X-linked recessive disorders of sex development described for the first time in 1953 by John Morris. These conditions are characterized by partial or complete inability of the cell to respond to androgen secondary to mutation in the Androgen Receptor gene located on long arm of the $X$ chromosome (locus X q 11-12) in the form of complete or partial deletions, point mutations or small insertions. ${ }^{1}$ The unresponsiveness of the cell to the presence of androgen hormones can impair or prevent the masculinisation of male genitalia, development of male secondary sexual characters with no significant impairment of female sexual development occurring only in genetic XY male. Although the external genitalia are female, development of internal female organs is suppressed by Mullerian inhibiting factor from the fetal testes.

Androgen insensitivity syndrome is divided into three categories: Complete AIS is indicated when the external genitalia are that of a normal female; Mild AIS, when the external genitalia are that of a normal male and Partial AIS when the external genitalia are partially but not fully masculinised.

Another supplemental seven phenotypic grading was proposed by A Quigley et al in $1995 .^{3}$ Grade one is when the external genitalia is fully masculinised, grade six is indicated with fully feminized external genitalia with grades two through five quantify four degree of decreasingly masculinisation lying in the interim. Grade seven is differentiated from grade six only after puberty with the absence of secondary terminal hairs.

The incidence of complete androgen insensitivity syndrome (CAIS) is 1:20000-64000/male birth. These are phenotypically female and are almost always raised as a girl. These subjects present with either primary amenorrhea or inguinal hernia in an apparently female infant with $46 \mathrm{XY}$ genotype. At puberty breast development occurs but menstruation and pubic-axillary hair growth does not occur. Imaging modality will confirm absent uterus, ovaries, short blind ending vagina and localization of testis. Our first case was diagnosed incidentally where she presented with right inguinal hernia with USG and MRI demonstrating Mullerian agenesis, bilateral inguinal testis and right inguinal hernia containing omentum.
Partial androgen insensitivity syndrome (PAIS) is diagnosed when the degree of androgen insensitivity in a $46 \mathrm{XY}$ karyotype individual is great enough to partially prevent the masculinisation of the genitalia. Patient present with either ambiguous genitalia, primary amenorrhea or with inguinal hernia.

Given the wide diversity of phenotypes, the diagnosis of PAIS is specified by assessing genital masculinisation using Quigley scale of grade two through grade five.

Grade 2 PAIS, the mildest form present with male phenotype but with small penis and hypospadias.

Grade 3 PAIS have predominantly male phenotype with micropenis, perineal hypospadias, cryptorchidism and possibly bifid scrotum.

Grade 4 PAIS present with a gender ambiguous phenotype with clitoromegaly, urogenital sinus, perineal opening and labioscrotal folds.

Grade 5 PAIS is predominantly female phenotype with large clitoris, separate opening of urethra and vagina.

Quigley et al draw attention to the question of pubic, axillary hairs in androgen insensitivity syndrome and says that the presence of true pubic hairs even in an individual with an entirely female phenotype, must be regarded as evidence for some degree of androgen responsiveness and that such individuals should be considered as having a severe partial form androgen insensitivity rather than complete androgen insensitivity. Mild androgen insensitivity syndrome present in a normal male phenotype which are usually undiagnosed, may present only with male infertility with oligo-azoospermia, reduced secondary pilosity.

The hormonal assay in both CAIS, PAIS are same with normal or elevated serum testosterone. The increased testosterone is peripherally aromatised to oestrogen which together with LH induced direct secretion of testicular oestrogen, results in increased serum oestradiol concentration responsible for adequate breast development at puberty in complete androgen insensitivity syndrome. In patient with partial androgen insensitivity syndrome quantification of testosterone, dihydro testosterone, T/DHT ratio and post beta HCG stimulation T/DHT ratio is important to exclude defects in testosterone biosynthesis and 5 alpha reductase deficiencies. ${ }^{4}$

The ectopic testis carries a overall risk of malignancy of $5-10 \%$ comprising of seminoma, dysgerminoma arising from the premalignant precursor called carcinoma in situ or intratubular germ cell neoplasia. Gonadal malignancy risk is even higher in partial androgen insensitivity syndrome with incidence of $15 \%$ or higher. ${ }^{5}$ Radiological imaging plays an important part in diagnosis and management. ${ }^{6}$ Ultrasonography is first line of 
investigation to confirm Mullerian agenesis and location of testis especially at the level of inguinal canal and labia. MRI is gold standard for diagnosis of Mullerian agenesis, finding wolffian remnants, evaluation of blind ending vagina, abdominal and pelvic localization of testes as well as development of any gonadal malignancy. ${ }^{7}$ Role of imaging cannot be ignored in cases with atypical presentation like with inguinal- labial swelling/hernias and also in the planning for laproscopic gonadectomy.

Management of androgen insensitivity syndrome needs multidisciplinary approach emphasing functional, sexual and psychological issues. Disclosure must be done carefully addressing issues like implications of an XY karyotype, presence of testis, absent uterus, sexual function, starting with parents initially with age appropriate disclosure to patients. ${ }^{8}$

Complete androgen insensitivity presenting in infancy needs early gonadectomy with puberty induction with oestrogen replacement. Gonadectomy can be delayed until early adulthood. ${ }^{9}$ Vaginal surgery is rarely indicated with trained vaginal dilatation suffice the sexual function.

Sex assignment and early management issues are more important in partial androgen insensitivity syndrome as they usually present with ambiguous genitalia. ${ }^{8}$ Most infants are raised as male. Surgery is done in second to third years of life for hypospadias and to bring undescended testes into scrotum. An infant who is assigned female will need a genitoplasty and gonadectomy before puberty to avoid virilisation. Oestrogen replacement is needed for puberty induction and thereafter for proper bone mineralisation.

\section{CONCLUSION}

Androgen insensitivity syndrome is one of the commonest examples of disorder of sex development which requires utmost sensitivity and multidisciplinary approach. This disorder should be considered in patients presenting with amenorrhea, inguinal hernia or variation in external genitalia appearance with imaging playing important role in identifying the ectopic testis. Sensitive disclosure is needed to allow young female and families to make informed decision about treatment and a realistic adaptation to life.

Funding: No funding sources

\section{Conflict of interest: None declared Ethical approval: Not required}

\section{REFERENCES}

1. Galani A, Kitsiou-Tzeli S, Sofokleous C, Kanavakis E, Kalpini-Mavrou A. Androgen insensitivity syndrome: clinical features and molecular defects. Hormones (Athens). 2008;7(3):217-29.

2. Horterhus P, Werner R, Hoppe U, Bassler J, Korsch E, et al. Molecular defects and clinical phenotypes in androgen insensitivity syndrome in the absence and presence of androgen receptor gene mutations. J Mol Med. 2005;83(12):1005-13.

3. Quigley CA, De Bellis A, Marschke KB, el-Awady MK, Wilson EM, et al. Androgen receptor defects: histological, clinical and molecular perspectives. Endocr Rev. 1995;16(3):271-321.

4. Veiga-Junior NN, Medaets PA, Petroli RJ, Calais FL, de Mello MP, et al. Clinical and laboratorial features that may differentiate $46, \mathrm{XY}$ DSD due to partial androgen insensitivity and 5 alpha reductase type 2 deficiency. Int $\mathrm{J}$ Endocriol. 2012;2012:964876.

5. Liu AX, Shi HY, Cai ZJ, Liu A, Zhang D, et al. Increased risk of gonadal malignancy and prophylactic gonadectomy: a study of 102 pheotypic female patients with $\mathrm{Y}$ chromosome or Y-derived sequences. Human Reprod. 2014;29(7):1413-9.

6. Khan S, Craig LTB. A review of radiologic imaging in patients with androgen insensitivity. J Genit Syst Disor. 2013:S1.

7. Nezzo M, De Visschere P, T'sjoen G, Weyers S, Villeirs G. Role of imaging in the diagnosis and management of complete androgen insensitivity syndrome in adults. Case Rep Radiol. 2013;2013:158484.

8. Hughes IA, Houk C, Ahmed SF, Lee PA. Consensus statement on management of intersex disorders. Arch Dis Child. 2006;91(7):554-63.

9. Kravarusic D, Sequier-Lipszyc E, Feigin E, Nimri. Androgen insensitivity syndrome: risk of malignancy and timing of surgery in a pediatric and adolescent population. Afr J Pediatr Surg. 2011;8(2):1.

Cite this article as: Sharma G, Ghode R. Androgen insensitivity syndrome: discussion based on three cases. Int J Reprod Contracept Obstet Gynecol 2015;4:1585-8. 